\title{
Fatores preditores do diagnóstico de enfermagem padrão respiratório ineficaz em pacientes de uma unidade de terapia intensiva*
}

\author{
Patricia Rezende do Prado ${ }^{1,2}$ \\ (D) https://orcid.org/0000-0002-3563-6602 \\ Ana Rita de Cássia Bettencourt ${ }^{3}$ \\ (D) https://orcid.org/0000-0002-4346-6586 \\ Juliana de Lima Lopes ${ }^{3}$ \\ (D) https://orcid.org/0000-0001-5779-1358
}

Objetivo: identificar os fatores preditores e a sensibilidade, especificidade, valores preditivos positivo e negativo do diagnóstico de enfermagem Padrão respiratório ineficaz em pacientes de uma unidade de terapia intensiva. Método: estudo transversal. Foi realizada a regressão logística para avaliar os efeitos simultâneos dos fatores relacionados. Resultados: dos 120 pacientes, 67,5\% apresentaram padrão respiratório ineficaz. Na análise univariada, os fatores relacionados foram: grupo de doenças, fadiga, obesidade e a secreção nos brônquios, e as características definidoras foram: alteração na profundidade respiratória, ausculta com ruídos adventícios, dispneia, murmúrios vesiculares diminuídos, taquipneia, tosse e uso da musculatura acessória para respirar. A média de idade dos pacientes com este diagnóstico foi superior ao daqueles sem este diagnóstico. A característica definidora murmúrios vesiculares diminuídos teve alta sensibilidade $(92,6 \%)$, especificidade $(97,4 \%)$, valor preditivo negativo $(86,4 \%)$ e valor preditivo positivo $(98,7 \%)$. Os fatores relacionados de padrão respiratório ineficaz foram a fadiga, a idade e o grupo de doenças. Conclusão: a fadiga, a idade e pacientes com grupo de doenças foram fatores preditores do diagnóstico padrão respiratório ineficaz neste estudo. Os murmúrios vesiculares diminuídos, a ausculta com ruídos adventícios e a tosse podem ser características definidoras a serem acrescentadas na classificação internacional, assim como os fatores relacionados secreção nos brônquios e grupo de doenças.

Descritores: Fatores de Risco; Sinais e Sintomas; Diagnóstico de Enfermagem; Sistema Respiratório; Classificação; Enfermagem.

\section{Como citar este artigo}

Prado PR, Bettencourt ARC, Lopes JL. Related factors of the nursing diagnosis ineffective breathing pattern in an intensive care unit. Rev. Latino-Am. Enfermagem. 2019;27:e3153. [Access DOI: http://dx.doi.org/10.1590/1518-8345.2902.3153.

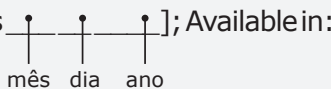




\section{Introdução}

A avaliação do padrão respiratório é essencial para definir as intervenções de enfermagem e estabelecer o plano de cuidados que atendam às necessidades do paciente. Esta avaliação deve ser realizada por meio do exame físico com monitoramento das funções fisiológicas da inspeção torácica, palpação, percussão e ausculta pulmonar, as quais fornecem dados objetivos do uso dos músculos respiratórios, da frequência respiratória e dos sons pulmonares ${ }^{(1-2)}$.

Em condições normais, o padrão respiratório satisfaz a necessidade de oxigenação do corpo, no entanto, em situações em que exista fadiga, comprometimento das vias aéreas devido à secreção e diminuição da expansão pulmonar caracteriza o diagnóstico de enfermagem (DE) padrão respiratório ineficaz (PRI) $)^{(3)}$

O DE padrão de respiração ineficaz (PRI) foi definido pela primeira vez em 1980 e revisado em 1996, 1998, 2010 e 2017. Em 2017, esse diagnóstico foi modificado e incluiu as condições associadas. É um diagnóstico com foco no problema e pertence ao domínio 4, classe 4, atividade/repouso da NANDA International, Inc. (NANDA-I). PRI é definido como um padrão de inspiração e/ou expiração que não fornece ventilação adequada(4).

Esse diagnóstico tem sido identificado frequentemente em indivíduos adultos e em diversas unidades. Em pacientes vítimas de trauma atendidos em um hospital universitário da cidade de São Paulo, SP, Brasil, observou-se que $82,4 \%$ apresentaram PRI ${ }^{(5)}$ e $85.7 \%$ em adultos em salas de emergência(6). Em pacientes adultos com cardiopatia, observou-se que este diagnóstico estava presente em $70,6 \%$ e que $100,0 \%$ deles apresentaram a fadiga como fator relacionado (FR) e a dispneia como característica definidora $(C D)^{(7)}$.

A primeira pesquisa americana identificou 0 diagnóstico PRI em $81,0 \%$ dos pacientes em unidade de terapia intensiva ${ }^{(8)}$. Na cidade de Rio Branco, Acre (AC), foi identificada prevalência de $64,4 \%$ de PRI em pacientes internados em UTI ${ }^{(9)}$, no entanto, esses estudos avaliaram apenas a prevalência, não identificaram as medidas de acurácia e também não avaliaram os fatores preditores do diagnóstico de enfermagem PRI. Assim, observa-se que o diagnóstico PRI é muito frequente na UTI. Devido à importância da identificação precoce e do estabelecimento de um plano de cuidados para esses pacientes, o objetivo deste estudo foi identificar os fatores preditores e a sensibilidade, especificidade, o valor preditivo positivo e negativo do diagnóstico de enfermagem Padrão Respiratório Ineficaz de pacientes de uma Unidade de Terapia Intensiva.

\section{Método}

Este é um estudo observacional, transversal, analítico. A pesquisa foi realizada na UTI do Hospital de Urgência e Emergência da cidade de Rio Branco, AC, Brasil, de setembro de 2015 a abril de 2016. Calculou-se o tamanho da amostra usando a fórmula para populações finitas, usando um coeficiente de $95 \%$ de confiança; um erro aleatório de $5 \%$; prevalência de PRI de $64,4 \%$, de acordo com um estudo realizado em uma UTI da cidade

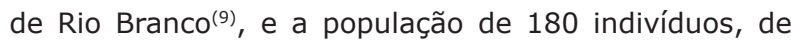
acordo com o número de pacientes conscientes e orientados hospitalizados nesta unidade pelo período de um ano. Esse perfil de paciente considerou a necessidade de realizar o teste de manovacuometria. Assim, o tamanho da amostra foi de 109 pacientes. Considerando $10 \%$ de probabilidade de perda, foram coletados dados de 120 pacientes.

Os critérios de inclusão foram: pacientes adultos acima de 18 anos, conscientes e orientados, sem doença neuromuscular, identificados pela avaliação médica e de enfermagem e com prontuário, com respiração espontânea, que aceitaram e puderam realizar o teste de manovacuometria. Foram excluídos pacientes com instabilidade hemodinâmica que pudessem interferir no teste de manovacuometria(10-11).

As variáveis independentes do estudo ( $C D, F R$ e condições associadas) foram identificadas na NANDA-I, taxonomia de classificação para o diagnóstico de Enfermagem $\mathrm{PRI}^{(4)}$ e em revisão da literatura (idade, tabagismo, grupo de doenças, secreção brônquica, tosse, murmúrios vesiculares diminuídos e ausculta com ruídos adventícios) ${ }^{(12)}$. Essas variáveis selecionadas foram avaliadas apenas pelo investigador principal do estudo por meio de entrevistas e exames físicos nos leitos dos pacientes, de acordo com a definição conceitual e operacional de cada variável, na qual foram previamente validadas(13-15) e outros foram adaptadas para a população adulta, como a assumir posição de três pontos para respirar e os valores de bradipneia/ taquipneia para a população adulta. As definições conceituais e operacionais das variáveis que não foram validadas em outros estudos foram elaboradas pelos pesquisadores, conforme a literatura(2,16-21).

Todos as CDs e FRs foram categorizados como presentes ou ausentes somente pelo investigador principal, exceto o grupo de doenças que foram categorizados de acordo com o grupo diagnosticado pelo médico. Os principais grupos de doenças identificados foram o trauma (ferida causada por arma de fogo e outras armas, fratura do fêmur e lesão cerebral traumática); doenças cardiocirculatórias (infarto agudo do miocárdio, bloqueio atrioventricular e acidente vascular cerebral isquêmico ou hemorrágico); doenças do sistema 
respiratório (edema pulmonar agudo e pneumonia); e outros grupos de doenças (abdómen agudo, sepse, picada de cobra, afogamento, convulsões, intoxicação exógena, laparotomia exploradora, lúpus eritematoso sistémico, leptospirose, pancreatite e hipertensão específica da gravidez).

As características definidoras avaliadas de PRI foram: mudanças na profundidade da respiração, assumir posição de três pontos para respirar, batimentos da asa de nariz, bradipneia, aumento do diâmetro anteroposterior do tórax, diminuição da pressão inspiratória, diminuição da pressão expiratória, dispneia, alteração da excursão torácica, prolongamento da fase de expiração, ortopneia, padrão respiratório anormal, respiração com lábios franzidos, taquipneia, uso de musculatura acessória para respirar, diminuição da ventilação em um minuto, tosse ${ }^{(12)}$, ausculta com ruídos adventícios $^{(12)}$ e murmúrios vesiculares diminuídos ${ }^{(4,12)}$.

Os fatores relacionados de PRI avaliados foram: ansiedade, dor, fadiga, fadiga da musculatura respiratória, hiperventilação, obesidade, posição do corpo que impede a expansão pulmonar e a secreção brônquica(4,12). A idade, o tabagismo e o grupo de doenças também foram incluídos ${ }^{(12)}$.

As condições associadas de PRI avaliadas foram: deformidade da parede torácica, deformidade óssea, lesão musculoesquelética e síndrome da hipoventilação(4). As condições associadas como dano neurológico, imaturidade neurológica, lesão da medula espinal e disfunção neuromuscular foram excluídas porque nestas situações os pacientes não podiam realizar o teste de manovacuometria e foi um critério de exclusão neste estudo. A CD capacidade vital diminuída não foi avaliada porque não havia ventilador ou espirômetro na unidade, o que dificultou a avaliação da porcentagem máxima de gás exalado após inspiração. Estes foram fatores limitantes do estudo. A variável de desfecho (dependente) estudada foi a presença do diagnóstico de enfermagem do PRI, definido como "inspiração e/ou expiração que não proporciona ventilação adequada"(4). Para esse diagnóstico, os pacientes deveriam ter três ou mais CDs e a pressão inspiratória máxima ser menor que $80 \mathrm{cmH}_{2} \mathrm{O}$ para homens e menor que $60 \mathrm{cmH}_{2} \mathrm{O}$ para mulheres ${ }^{(22)}$. A pressão inspiratória máxima foi obtida pelo teste de manovacuometria(10), que é uma maneira simples de medir as pressões respiratórias máximas e é uma medida quantitativa da função e força dos músculos respiratórios, o que indica se a ventilação é adequada.

A CD pressão inspiratória diminuída avaliada pelo manovacuômetro foi escolhida para confirmar a presença de PRI, pois um estudo realizado em 2015 e 2016, com 626 pacientes adultos em UTI mostrou que essa $C D$ e o FR fadiga foram os fatores que apresentaram maior sensibilidade para o diagnóstico PRI nesses pacientes ${ }^{(23)}$. A enfermeira responsável por esta pesquisa coletou os dados com a ajuda de um instrumento padronizado elaborado para esse fim. A coleta foi realizada todos os dias pela manhã com pacientes que atendiam aos critérios de inclusão na UTI. Após a coleta de dados, os pacientes que apresentavam e não apresentavam o diagnóstico de enfermagem do PRI foram comparados com a causalidade do diagnóstico de enfermagem PRI.

Para o teste de manovacuometria, os sujeitos foram avaliados em uma posição sentada $\left(90^{\circ}\right)$, utilizando um grampo de nariz com um embolo de borracha semirrígido, com um orifício interno de $2 \mathrm{~mm}$ de diâmetro, em que o paciente foi solicitado para fechar os lábios firmemente ao redor do bocal. Para medir a pressão inspiratória máxima (PImax), o paciente foi solicitado a exalar o dispositivo, o qual foi ocluído e, em seguida, fez-se um esforço inspiratório contra a via aérea ocluída registrando-se assim, a PImax. Os pacientes mantiveram a pressão inspiratória por pelo menos 1,5 segundos e a maior pressão negativa sustentada foi registrada. Esse mesmo processo foi repetido três vezes, com intervalos de um minuto em cada avaliação, sendo utilizado apenas o maior valor ${ }^{(11)}$. Ressalta-se que caso ocorresse diferenças de valores superiores a $10 \%$ obtidas entre as medidas, elas foram descartadas.

O manovacuômetro utilizado foi adquirido para uso único, do tipo analógico, fabricante Wika, modelo 611.10.063L, + 120/-120, calibrado de acordo com o procedimento interno PRP-04-re.13, NBR-ISO-10012, parte 1. Após a avaliação, os pacientes foram divididos em dois grupos, com e sem PRI.

Este projeto foi aprovado pelo Comitê de Ética em Pesquisa (CEP) da Universidade Federal de São Paulo (UNIFESP) sob o parecer número 1.290.590, CAAE: 39185814.9.0000.5505, em 21/10/2015. Esta pesquisa seguiu as diretrizes da Resolução do Conselho Nacional de Ética em Pesquisa (CONEP) no 466/2012, do Conselho Nacional de Saúde (CNS) do Brasil e todos os pacientes entrevistados foram informados e assinaram o Termo de Consentimento Livre e Esclarecido que autoriza a investigação.

Os dados foram analisados utilizando o pacote estatístico de ciências sociais (SPSS), Microsoft Office, Universidade de Chicago, versão 20.0. Uma análise descritiva inicial dos dados foi realizada. Frequências absolutas e relativas foram utilizadas para as variáveis categóricas e as medidas de resumo (média, quartis, mínimo, máximo e desvio padrão) para as variáveis numéricas.

A associação entre duas variáveis categóricas foi verificada pelo teste do qui-quadrado ou pelo teste 
exato de Fisher nos casos de amostras pequenas. Quando diferenças nas distribuições foram observadas, os resíduos ajustados padronizados foram usados para identificar as diferenças locais. A comparação de médias entre dois grupos foi realizada utilizando o teste $t$ de Student para amostras independentes.

Para todas as características definidoras e fatores relacionados de natureza dicotômica, foram apresentadas medidas de acurácia pela sensibilidade, especificidade, valor preditivo positivo (VPP) e valor preditivo negativo (VPN). As regressões logísticas foram ajustadas para avaliar os efeitos simultâneos dos FR na presença de PRI. Devido ao grande número de variáveis que predizem o tamanho da amostra, as variáveis cujas associações foram significativas a $20 \%$, entraram no modelo para a análise univariada. Em seguida, as variáveis não significativas a $5 \%$ foram excluídas uma a uma por ordem de importância (método backward).

O teste de Hosmer e Lemeshow foi utilizado para analisar o ajuste do modelo final, considerando o FR como variáveis relacionadas. A sensibilidade e a especificidade foram calculadas com base na curva ROC, o que permitiu a definição de um ponto de corte nas probabilidades de ocorrência de PRI estimado a partir do modelo de regressão final ajustado. Foi utilizado um nível de significância de 5\% para todos os testes estatísticos.

\section{Resultados}

Dos 120 pacientes da amostra, 30,0\% eram idosos, com média de idade de 47,0 anos, $60,8 \%$ era do sexo masculino, 59,2\% eram pardos e $61,7 \%$ tinham até o ensino fundamental de escolaridade. Os principais grupos de doenças identificados foram trauma (ferimento por arma de fogo e branca, fratura de fêmur e traumatismo crânio encefálico); doenças cardiocirculatórias (infarto agudo do miocárdio, bloqueio átrio ventricular e acidente vascular cerebral isquêmico ou hemorrágico); doenças do aparelho respiratório (edema agudo de pulmão e pneumonia) e outros grupos de doenças (abdome agudo, sepse, acidente ofídico, afogamento, convulsão intoxicação exógena, laparotomia exploratória, lúpus eritematoso sistêmico, leptospirose, pancreatite e doença hipertensiva específica da gravidez).

Dentre os pacientes avaliados, $67,5 \%$ apresentaram o diagnóstico de enfermagem PRI. Observa-se na Tabela 1 que PRI se associou com as seguintes CDs: alteração na profundidade respiratória, ausculta com ruídos adventícios, dispneia, murmúrio vesicular diminuído, taquipneia, tosse e uso da musculatura acessória para respirar. Nota-se que $100 \%$ dos pacientes com essas CDs, exceto murmúrio vesicular diminuído, apresentaram PRI.

$\mathrm{Na}$ tabela 2, observa-se a sensibilidade, especificidade e valores preditivos positivos $e$ negativos das CDs de PRI. Pode-se notar que apenas a $C D$ murmúrio vesicular diminuído apresentou alta sensibilidade $(92,6 \%)$, alta especificidade $(97,4 \%)$ e VPP e VPN acima de $86 \%$. A ausculta com ruídos adventícios, por sua vez, mostrou também alta sensibilidade $(71,6 \%)$, especificidade $(100,0 \%)$ e VPP $(100,0 \%)$, porém, VPN moderada $(62,9 \%)$. As demais características, exceto padrão respiratório anormal e pressão inspiratória diminuída, apresentaram altas especificidade, porém baixa sensibilidade. Padrão inverso foi observado para padrão respiratório anormal e pressão inspiratória máxima.

Tabela 1 - Características definidoras segundo a presença ou ausência do diagnóstico de enfermagem Padrão respiratório ineficaz. Rio Branco, AC, Brasil, 2015-2016

\begin{tabular}{|c|c|c|c|c|c|c|c|c|}
\hline & \multicolumn{4}{|c|}{ PRI } & \multirow{2}{*}{\multicolumn{2}{|c|}{ Total }} & \multirow{3}{*}{ RC } & \multirow{3}{*}{ p-valor* } \\
\hline & \multicolumn{2}{|c|}{ Ausente } & \multicolumn{2}{|c|}{ Presente } & & & & \\
\hline & $\mathbf{n}$ & $\%$ & n & $\%$ & $\mathbf{n}$ & $\%$ & & \\
\hline Alteração na profundidade respiratória & 39 & $32,5 \%$ & 81 & $67,5 \%$ & 120 & $100,0 \%$ & & $<0,001^{*}$ \\
\hline Ausente & 39 & $44,8 \%$ & 48 & $55,2 \%$ & 87 & $100,0 \%$ & 1,00 & \\
\hline Presente & 0 & $0,0 \%$ & 33 & $100,0 \%$ & 33 & $100,0 \%$ & $(1)^{\dagger}$ & \\
\hline Ausculta com ruídos adventícios & 39 & $32,5 \%$ & 81 & $67,5 \%$ & 120 & $100,0 \%$ & & $<0,001^{*}$ \\
\hline Ausente & 39 & $62,9 \%$ & 23 & $37,1 \%$ & 62 & $100,0 \%$ & 1,00 & \\
\hline Presente & 0 & $0,0 \%$ & 58 & $100,0 \%$ & 58 & $100,0 \%$ & $(1)^{\dagger}$ & \\
\hline Batimento de asa nariz & 39 & $32,5 \%$ & 81 & $67,5 \%$ & 120 & $100,0 \%$ & & $0,172^{\ddagger}$ \\
\hline Ausente & 39 & $33,9 \%$ & 76 & $66,1 \%$ & 115 & $100,0 \%$ & 1,00 & \\
\hline Presente & 0 & $0,0 \%$ & 5 & $100,0 \%$ & 5 & $100,0 \%$ & $(1)^{\dagger}$ & \\
\hline Bradipneia & 39 & $32,5 \%$ & 81 & $67,5 \%$ & 120 & $100,0 \%$ & & - \\
\hline Ausente & 39 & $32,5 \%$ & 81 & $67,5 \%$ & 120 & $100,0 \%$ & - & \\
\hline
\end{tabular}




\begin{tabular}{|c|c|c|c|c|c|c|c|c|}
\hline & \multicolumn{4}{|c|}{ PRI } & \multirow{2}{*}{\multicolumn{2}{|c|}{ Total }} & \multirow{3}{*}{ RC } & \multirow{3}{*}{$\mathrm{p}$-valor* } \\
\hline & \multicolumn{2}{|c|}{ Ausente } & \multicolumn{2}{|c|}{ Presente } & & & & \\
\hline & $\mathbf{n}$ & $\%$ & $\mathrm{n}$ & $\%$ & $\mathbf{n}$ & $\%$ & & \\
\hline Diâmetro anteroposterior aumentado & 39 & $32,5 \%$ & 81 & $67,5 \%$ & 120 & $100,0 \%$ & & $0,172^{\ddagger}$ \\
\hline Ausente & 39 & $33,9 \%$ & 76 & $66,1 \%$ & 115 & $100,0 \%$ & 1,00 & \\
\hline Presente & 0 & $0,0 \%$ & 5 & $100,0 \%$ & 5 & $100,0 \%$ & $(1)^{\dagger}$ & \\
\hline Dispneia & 39 & $32,5 \%$ & 81 & $67,5 \%$ & 120 & $100,0 \%$ & & $<0,001^{*}$ \\
\hline Ausente & 39 & $41,9 \%$ & 54 & $58,1 \%$ & 93 & $100,0 \%$ & 1,00 & \\
\hline Presente & 0 & $0,0 \%$ & 27 & $100,0 \%$ & 27 & $100,0 \%$ & $(1)^{\dagger}$ & \\
\hline Excursão torácica alterada & 39 & $32,5 \%$ & 81 & $67,5 \%$ & 120 & $100,0 \%$ & & $0,052^{\ddagger}$ \\
\hline Ausente & 39 & $34,8 \%$ & 73 & $65,2 \%$ & 112 & $100,0 \%$ & 1,00 & \\
\hline Presente & 0 & $0,0 \%$ & 8 & $100,0 \%$ & 8 & $100,0 \%$ & $(1)^{\dagger}$ & \\
\hline Padrão respiratório anormal & 39 & $32,5 \%$ & 81 & $67,5 \%$ & 120 & $100,0 \%$ & & $0,328^{\ddagger}$ \\
\hline Ausente & 3 & $60,0 \%$ & 2 & $40,0 \%$ & 5 & $100,0 \%$ & 1,00 & \\
\hline Presente & 36 & $31,3 \%$ & 79 & $68,7 \%$ & 115 & $100,0 \%$ & 3,29 & \\
\hline Fase de expiração prolongada & 39 & $32,5 \%$ & 81 & $67,5 \%$ & 120 & $100,0 \%$ & & $1,000^{\ddagger}$ \\
\hline Ausente & 39 & $32,8 \%$ & 80 & $67,2 \%$ & 119 & $100,0 \%$ & 1,00 & \\
\hline Presente & 0 & $0,0 \%$ & 1 & $100,0 \%$ & 1 & $100,0 \%$ & $(1)^{\dagger}$ & \\
\hline Respiração com lábios franzidos & 39 & $32,5 \%$ & 81 & $67,5 \%$ & 120 & $100,0 \%$ & & $1,000^{\ddagger}$ \\
\hline Ausente & 39 & $33,1 \%$ & 79 & $66,9 \%$ & 118 & $100,0 \%$ & 1,00 & \\
\hline Presente & 0 & $0,0 \%$ & 2 & $100,0 \%$ & 2 & $100,0 \%$ & $(1)^{\dagger}$ & \\
\hline Murmúrio vesicular diminuído & 39 & $32,5 \%$ & 81 & $67,5 \%$ & 120 & $100,0 \%$ & & $<0,001^{*}$ \\
\hline Ausente & 38 & $86,4 \%$ & 6 & $13,6 \%$ & 44 & $100,0 \%$ & 1,00 & \\
\hline Presente & 1 & $1,3 \%$ & 75 & $98,7 \%$ & 76 & $100,0 \%$ & 475,00 & \\
\hline Ortopneia & 39 & $32,5 \%$ & 81 & $67,5 \%$ & 120 & $100,0 \%$ & & $1,000^{\ddagger}$ \\
\hline Ausente & 39 & $32,8 \%$ & 80 & $67,2 \%$ & 119 & $100,0 \%$ & 1,00 & \\
\hline Presente & 0 & $0,0 \%$ & 1 & $100,0 \%$ & 1 & $100,0 \%$ & $(1)^{\dagger}$ & \\
\hline Pressão expiratória diminuída & 39 & $32,5 \%$ & 81 & $67,5 \%$ & 120 & $100,0 \%$ & & - \\
\hline Presente & 39 & $32,5 \%$ & 81 & $67,5 \%$ & 120 & $100,0 \%$ & - & \\
\hline Pressão inspiratória diminuída & 39 & $32,5 \%$ & 81 & $67,5 \%$ & 120 & $100,0 \%$ & & $0,325^{\ddagger}$ \\
\hline Ausente & 1 & $100,0 \%$ & 0 & $0,0 \%$ & 1 & $100,0 \%$ & $(2)^{\S}$ & \\
\hline Presente & 38 & $31,9 \%$ & 81 & $68,1 \%$ & 119 & $100,0 \%$ & & \\
\hline Assumir posição de três pontos & 39 & $32,5 \%$ & 81 & $67,5 \%$ & 120 & $100,0 \%$ & & $0,172^{\ddagger}$ \\
\hline Ausente & 39 & $33,9 \%$ & 76 & $66,1 \%$ & 115 & $100,0 \%$ & 1,00 & \\
\hline Presente & 0 & $0,0 \%$ & 5 & $100,0 \%$ & 5 & $100,0 \%$ & $(1)^{\dagger}$ & \\
\hline Taquipneia & 39 & $32,5 \%$ & 81 & $67,5 \%$ & 120 & $100,0 \%$ & & $<0,001^{*}$ \\
\hline Ausente & 39 & $40,2 \%$ & 58 & $59,8 \%$ & 97 & $100,0 \%$ & 1,00 & \\
\hline Presente & 0 & $0,0 \%$ & 23 & $100,0 \%$ & 23 & $100,0 \%$ & $(1)^{\dagger}$ & \\
\hline Tosse & 39 & $32,5 \%$ & 81 & $67,5 \%$ & 120 & $100,0 \%$ & & $0,001^{*}$ \\
\hline Ausente & 39 & $40,6 \%$ & 57 & $59,4 \%$ & 96 & $100,0 \%$ & 1,00 & \\
\hline Presente & 0 & $0,0 \%$ & 24 & $100,0 \%$ & 24 & $100,0 \%$ & $(1)^{\dagger}$ & \\
\hline Uso da musculatura acessória para respirar & 39 & $32,5 \%$ & 81 & $67,5 \%$ & 120 & $100,0 \%$ & & $<0,001^{*}$ \\
\hline Ausente & 39 & $39,0 \%$ & 61 & $61,0 \%$ & 100 & $100,0 \%$ & 1,00 & \\
\hline Presente & 0 & $0,0 \%$ & 20 & $100,0 \%$ & 20 & $100,0 \%$ & $(1)^{\dagger}$ & \\
\hline Ventilação minuto diminuída & 39 & $32,5 \%$ & 81 & $67,5 \%$ & 120 & $100,0 \%$ & & $1,000^{\ddagger}$ \\
\hline Ausente & 39 & $33,1 \%$ & 79 & $66,9 \%$ & 118 & $100,0 \%$ & 1,00 & \\
\hline Presente & 0 & $0,0 \%$ & 2 & $100,0 \%$ & 2 & $100,0 \%$ & $(1)^{\dagger}$ & \\
\hline
\end{tabular}


Tabela 2 - Sensibilidade, especificidade e valores preditivos positivos e negativos das características definidoras de Padrão respiratório ineficaz. Rio Branco, AC, Brasil, 2015-2016

\begin{tabular}{lcccc}
\hline \multirow{2}{*}{ Características definidoras } & Sensibilidade (\%) & Especificidade (\%) & \multicolumn{2}{c}{ Valor Preditivo (\%) } \\
\cline { 4 - 5 } & & & Positivo (VPP) & Negativo (VPN) \\
\hline Murmúrio vesicular diminuído & $92,6(84,6-97,2)$ & $97,4(86,5-99,9)$ & $98,7(92,9-100,0)$ & $86,4(72,6-94,8)$ \\
Ausculta com ruídos adventícios & $71,6(60,5-81,1)$ & $100,0(91,0-100,0)$ & $100(93,8-100,0)$ & $62,9(49,7-74,8)$ \\
Padrão respiratório anormal & $97,5(91,4-99,7)$ & $7,7(1,6-20,9)$ & $68,7(59,4-77,0)$ & $60,0(14,7-94,7)$ \\
Pressão inspiratória diminuída & $100,0(95,5-100)$ & $2,6(0,1-13,5)$ & $68,1(58,9-76,3)$ & $100,0(2,5-100,0)$ \\
Pressão expiratória diminuída & $100,0(-)$ & $0,0(-)^{*}$ & $67,5(-)$ & - \\
Alteração na profundidade respiratória & $40,7(29,9-52,2)$ & $100,0(91,0-100,0)$ & $100(89,4-100,0)$ & $44,8(34,1-55,9)$ \\
Dispneia & $33,3(23,2-44,7)$ & $100,0(91,0-100,0)$ & $100,0(87,2-100,0)$ & $41,9(31,8-52,6)$ \\
Tosse & $29,6(20,0-40,8)$ & $100,0(91,0-100,0)$ & $100(85,8-100,0)$ & $40,6(30,7-51,1)$ \\
Taquipneia & $28,4(18,9-39,5)$ & $100,0(91,0-100,0)$ & $100,0(85,2-100,0)$ & $40,2(30,4-50,7)$ \\
Uso da musculatura acessória para & $24,7(15,8-35,5)$ & $100,0(91,0-100,0)$ & $100,0(83,2-100,0)$ & $39,0(29,4-49,3)$ \\
respirar & $9,9(4,4-18,5)$ & $100,0(91,0-100,0)$ & $100,0(63,1-100,0)$ & $34,8(26,1-44,4)$ \\
Excursão torácica alterada & $6,2(2,0-13,8)$ & $100,0(91,0-100,0)$ & $100,0(47,8-100,0)$ & $33,9(25,3-43,3)$ \\
Diâmetro anteroposterior aumentado & $6,2(2,0-13,8)$ & $100,0(91,0-100,0)$ & $100,0(47,8-100,0)$ & $33,9(25,3-43,3)$ \\
Batimento de asa de nariz & $6,2(2,0-13,8)$ & $100,0(91,0-100,0)$ & $100,0(47,8-100,0)$ & $33,9(25,3-43,3)$ \\
Assumir posição de três pontos & $2,5(0,3-8,6)$ & $100,0(91,0-100,0)$ & $100,0(15,8-100,0)$ & $33,1(24,7-42,3)$ \\
Ventilação minuto diminuída & $2,5(0,3-8,6)$ & $100,0(91,0-100,0)$ & $100,0(15,8-100,0)$ & $33,1(24,7-42,3)$ \\
Lábios franzidos & $1,2(0,0-6,7)$ & $100,0(91,0-100,0)$ & $100,0(2,5-100,0)$ & $32,8(24,4-42,0)$ \\
Ortopneia & $1,2(0,0-6,7)$ & $100,0(91,0-100,0)$ & $100,0(2,5-100,0)$ & $32,8(24,4-42,0)$ \\
Fase de expiração prolongada & $0,0(-)^{*}$ & $100,0(-)^{*}$ & & $32,5(-)^{*}$ \\
Bradipneia & & & &
\end{tabular}

*(-) não foi possível calcular $=$ a característica definidora não apresentou um dos níveis

A Tabela 3 mostra os FR do diagnóstico de enfermagem PRI em ambos os grupos, com e sem o diagnóstico PRI. Os FRs que se associaram com PRI foram grupo de doenças, fadiga, obesidade e secreção brônquica. Dessa forma, pacientes com fadiga, obesidade e secreção brônquica apresentaram porcentagens maiores de PRI comparativamente àqueles sem essas condições. Por outro lado, os pacientes diagnosticados com problemas cardiocirculatórios, respiratórios e outros grupos de doenças apresentaram porcentagens menores de ocorrência de PRI comparativamente àqueles diagnosticados com trauma. A média de idade dos pacientes com PRI foi maior do que naqueles sem PRI. As condições relacionadas ao dano musculoesquelético, deformidade da parede torácica, deformidade óssea e síndrome de hipoventilação estiveram presentes em $67,5 \%$ dos pacientes e não foram estatisticamente significantes entre os grupos (valor de $p>0,05$ ).

Tabela 3 - Fatores relacionados segundo a presença ou ausência do diagnóstico de enfermagem Padrão respiratório ineficaz. Rio Branco, AC, Brasil, 2015-2016

\begin{tabular}{|c|c|c|c|c|c|c|c|c|}
\hline \multirow{3}{*}{ Fator relacionado } & \multicolumn{4}{|c|}{ Padrão respiratório ineficaz } & \multirow{2}{*}{\multicolumn{2}{|c|}{ Total }} & \multirow{3}{*}{$\begin{array}{l}\text { Razão de } \\
\text { chance }\end{array}$} & \multirow{3}{*}{ p-valor } \\
\hline & \multicolumn{2}{|c|}{ Ausente } & \multicolumn{2}{|c|}{ Presente } & & & & \\
\hline & $\mathbf{n}$ & $\%$ & $\mathbf{n}$ & $\%$ & $\mathbf{N}$ & $\%$ & & \\
\hline Ansiedade & 39 & $32,5 \%$ & 81 & $67,5 \%$ & 120 & $100,0 \%$ & & $0.272^{*}$ \\
\hline Ausente & 38 & $33,9 \%$ & 74 & $66,1 \%$ & 112 & $100,0 \%$ & 1.00 & \\
\hline Presente & 1 & $12,5 \%$ & 7 & $87,5 \%$ & 8 & $100,0 \%$ & 3.59 & \\
\hline Grupo de doenças & 39 & $32,5 \%$ & 81 & $67,5 \%$ & 120 & $100,0 \%$ & & $0.008^{\dagger}$ \\
\hline Trauma & 6 & $16,7 \%$ & 30 & $83,3 \%$ & 36 & $100,0 \%$ & 1.00 & \\
\hline Cardiocirculatório & 13 & $37,1 \%$ & 22 & $62,9 \%$ & 35 & $100,0 \%$ & 0.34 & \\
\hline Respiratório & 5 & $22,7 \%$ & 17 & $77,3 \%$ & 22 & $100,0 \%$ & 0.68 & \\
\hline Outros & 15 & $55,6 \%$ & 12 & $44,4 \%$ & 27 & $100,0 \%$ & 0.16 & \\
\hline Dor & 39 & $32,5 \%$ & 81 & $67,5 \%$ & 120 & $100,0 \%$ & & $0.058^{*}$ \\
\hline Ausente & 38 & $35,5 \%$ & 69 & $64,5 \%$ & 107 & $100,0 \%$ & 1.00 & \\
\hline Presente & 1 & $7,7 \%$ & 12 & $92,3 \%$ & 13 & $100,0 \%$ & 6.61 & \\
\hline
\end{tabular}


Tabela 3 - continuação

\begin{tabular}{|c|c|c|c|c|c|c|c|c|}
\hline \multirow{3}{*}{ Fator relacionado } & \multicolumn{4}{|c|}{ Padrão respiratório ineficaz } & \multirow{2}{*}{\multicolumn{2}{|c|}{ Total }} & \multirow{3}{*}{$\begin{array}{l}\text { Razão de } \\
\text { chance }\end{array}$} & \multirow{3}{*}{ p-valor } \\
\hline & \multicolumn{2}{|c|}{ Ausente } & \multicolumn{2}{|c|}{ Presente } & & & & \\
\hline & $\mathbf{n}$ & $\%$ & $\mathbf{n}$ & $\%$ & $\mathbf{N}$ & $\%$ & & \\
\hline Fadiga & 39 & $32,5 \%$ & 81 & $67,5 \%$ & 120 & $100,0 \%$ & & $<0.001^{\dagger}$ \\
\hline Ausente & 38 & $48,7 \%$ & 40 & $51,3 \%$ & 78 & $100,0 \%$ & 1.00 & \\
\hline Presente & 1 & $2,4 \%$ & 41 & $97,6 \%$ & 42 & $100,0 \%$ & 38.95 & \\
\hline $\begin{array}{l}\text { Fadiga da musculatura } \\
\text { respiratória }\end{array}$ & 39 & $32,5 \%$ & 81 & $67,5 \%$ & 120 & $100,0 \%$ & & $1.000^{*}$ \\
\hline Ausente & 39 & $33,1 \%$ & 79 & $66,9 \%$ & 118 & $100,0 \%$ & 1.00 & \\
\hline Presente & 0 & $0,0 \%$ & 2 & $100,0 \%$ & 2 & $100,0 \%$ & $(1)^{\dagger}$ & \\
\hline Hiperventilação & 39 & $32,5 \%$ & 81 & $67,5 \%$ & 120 & $100,0 \%$ & & $0.172^{*}$ \\
\hline Ausente & 39 & $33,9 \%$ & 76 & $66,1 \%$ & 115 & $100,0 \%$ & 1.00 & \\
\hline Presente & 0 & $0,0 \%$ & 5 & $100,0 \%$ & 5 & $100,0 \%$ & $(1)^{\dagger}$ & \\
\hline Obesidade & 39 & $32,5 \%$ & 81 & $67,5 \%$ & 120 & $100,0 \%$ & & $0.019^{*}$ \\
\hline Ausente & 35 & $38,0 \%$ & 57 & $62,0 \%$ & 92 & $100,0 \%$ & 1.00 & \\
\hline Presente & 4 & $14,3 \%$ & 24 & $85,7 \%$ & 28 & $100,0 \%$ & 3.68 & \\
\hline $\begin{array}{l}\text { Posição do corpo que prejudice } \\
\text { expansão pulmonar }\end{array}$ & 39 & $32,5 \%$ & 81 & $67,5 \%$ & 120 & $100,0 \%$ & & $0.550^{*}$ \\
\hline Ausente & 39 & $33,3 \%$ & 78 & $66,7 \%$ & 117 & $100,0 \%$ & 1.00 & \\
\hline Presente & 0 & $0,0 \%$ & 3 & $100,0 \%$ & 3 & $100,0 \%$ & $(1)^{\dagger}$ & \\
\hline Secreção brônquica & 39 & $32,5 \%$ & 81 & $67,5 \%$ & 120 & $100,0 \%$ & & $0.016^{*}$ \\
\hline Ausente & 39 & $35,8 \%$ & 70 & $64,2 \%$ & 109 & $100,0 \%$ & 1.00 & \\
\hline Presente & 0 & $0,0 \%$ & 11 & $100,0 \%$ & 11 & $100,0 \%$ & $(1)^{\dagger}$ & \\
\hline Tabagismo & 39 & $32,5 \%$ & 81 & $67,5 \%$ & 120 & $100,0 \%$ & & $0.155^{\star}$ \\
\hline Não & 27 & $38,0 \%$ & 44 & $62,0 \%$ & 71 & $100,0 \%$ & 1.00 & \\
\hline Sim & 8 & $32,0 \%$ & 17 & $68,0 \%$ & 25 & $100,0 \%$ & 1.30 & \\
\hline Ex-tabagista & 4 & $16,7 \%$ & 20 & $83,3 \%$ & 24 & $100,0 \%$ & 3.07 & \\
\hline
\end{tabular}

A Tabela 4 mostra os modelos de regressão logística univariada e multivariada. Observou-se que pacientes com fadiga apresentaram uma possibilidade de ter PRI 61,96 vezes mais do que aqueles sem fadiga. Por outro lado, observou-se que pacientes com doenças cardiocirculatórias e outros tipos de doenças tinham, respectivamente, $93 \%$ e $85 \%$ menos chances de ter PRI do que aqueles com diagnóstico de trauma. Observou-se também que, com o aumento de um ano de idade, a probabilidade de PRI aumenta em $6 \%$.

Table 4 - Regressão logística com modelo final dos fatores preditores do diagnóstico de enfermagem Padrão respiratório ineficaz. Rio Branco, AC, Brasil, 2015-2016

\begin{tabular}{lcccc}
\hline & Modelo univariado & & Modelo final multivariado \\
\cline { 2 - 5 } & Razão de chance bruta (95\%) & p-valor & Razão de chance ajustada (95\%) & p-valor \\
\hline Ansiedade & $3.59(0.43-30.29)$ & 0.239 & - & - \\
Deformidade na parede do tórax & $(1)^{\dagger}$ & 0.999 & - & - \\
Deformidade óssea & $(2)^{\dagger}$ & - & - & - \\
Dor & $6.61(0.83-52.8)$ & 0.075 & - & - \\
Fatiga & $38.95(5.10-297.4)$ & $<0.001$ & $61.96(6.88-557.74)$ & $<0.001$ \\
Fadiga da musculatura acessória & $(1)^{\dagger}$ & 0.999 & - & - \\
Hiperventilação & $(1)^{\dagger}$ & 0.999 & - & - \\
Obesidade & $3.68(1.18-11.51)$ & 0.025 & $2.76(0.64-11.84)$ & 0.171 \\
Posição no corpo que inibe a expansão & $(1)^{\dagger}$ & 0.999 & - & - \\
pulmonar & $3.04(0.35-26.17)$ & 0.311 & - & - \\
Dano musculoesquelético & $(1)^{\dagger}$ & 1.000 & - \\
Síndrome da hipoventilação & & & - \\
\hline
\end{tabular}


Tabela 4 - continuação

\begin{tabular}{|c|c|c|c|c|}
\hline & \multicolumn{2}{|l|}{ Modelo univariado } & \multicolumn{2}{|c|}{ Modelo final multivariado } \\
\hline & Razão de chance bruta (95\%) & p-valor* & Razão de chance ajustada (95\%) & p-valor* \\
\hline Secreção brônquica & $(1)^{\dagger}$ & 0.999 & - & - \\
\hline Idade (anos) & $1.03(1.01-1.05)$ & 0.009 & $1.06(1.02-1.09)$ & 0.001 \\
\hline Tabagismo (ref. = não) & & 0.172 & & \\
\hline Sim & $1.30(0.5-3.43)$ & 0.591 & & \\
\hline Ex-fumante & $3.07(0.95-9.94)$ & 0.062 & & \\
\hline Grupo de doenças (ref. = trauma) & & 0.011 & & 0.013 \\
\hline Cardiocirculatório & $0.34(0.11-1.03)$ & 0.056 & $0.07(0.01-0.38)$ & 0.002 \\
\hline Respiratório & $0.68(0.18-2.56)$ & 0.569 & $0.23(0.04-1.30)$ & 0.096 \\
\hline Outros & $0.16(0.05-0.51)$ & 0.002 & $0.15(0.04-0.62)$ & 0.009 \\
\hline
\end{tabular}

*Teste de Hosmer e Lemeshow para o melhor modelo final $(\mathrm{p}=0.649) ;{ }^{\dagger}(1)=$ ausência de casos do diagnóstico Padrão respiratório ineficaz na presença do fator relacionado; ${ }^{\ddagger}(2)$ = ausência de um dos níveis do fator relacionado

O modelo final com as informações dos fatores relacionados permite estimar a probabilidade de um paciente apresentar PRI. Utilizando a curva ROC, observamos uma boa capacidade relacionada das probabilidades de ocorrência de PRI estimado pelo modelo final (área sob a curva ROC 0.875) com alta sensibilidade $(82,72 \%)$ e especificidade (74,36\%) (Figura 1).

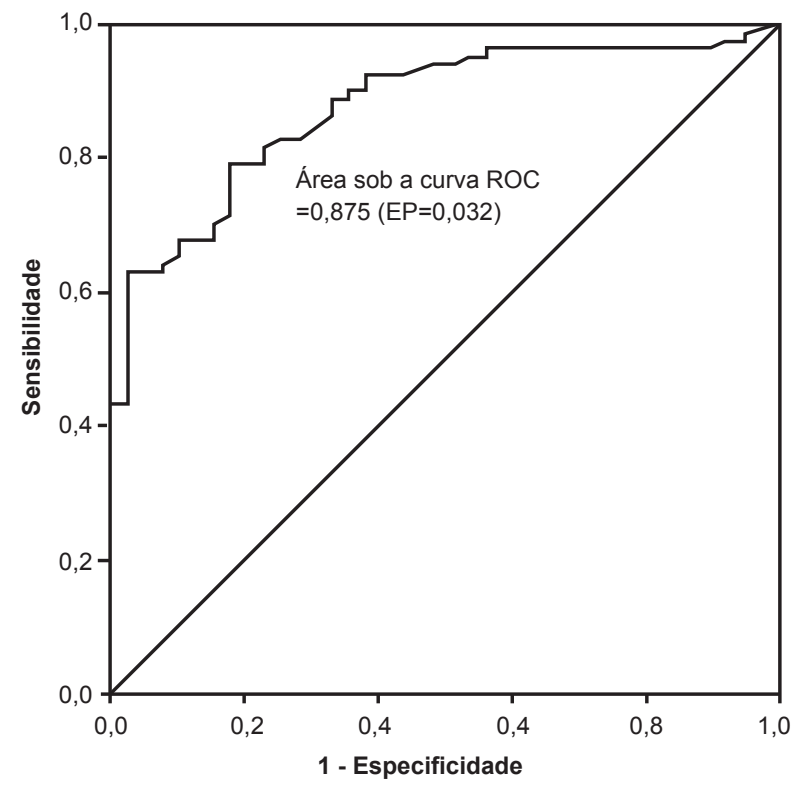

Figura 1 - Curva ROC para PRI. Rio Branco, AC, Brasil, 2015-2016

\section{Discussão}

Os fatores preditores do diagnóstico de enfermagem PRI nesta UTI foram os FR fadiga, idade e o grupo de doenças (sistema cardiocirculatório, trauma e outros diagnósticos). O desenvolvimento da fadiga é uma complicação importante e comum em muitos pacientes admitidos em UTI e sua incidência pode variar em torno de $30 \%$ a $60 \%$ nestes pacientes ${ }^{(11,24)}$. A fadiga tem sido avaliada em diversos estudos devido à sua alta prevalência e pelo prejuízo que causa à qualidade de vida dos pacientes ${ }^{(18,25-27)}$. Além das comorbidades prévias, vários fatores podem contribuir para a ocorrência da fadiga, incluindo inflamações sistêmicas, uso de alguns medicamentos como corticoides, sedativos e bloqueadores neuromusculares, desnutrição, hiperosmolaridade, nutrição parenteral, cardiopatias e imobilidade prolongada, condições comuns na UTI(25-26). A fadiga pode estar presente em pacientes com patologias diversas, como doença cardíaca, pulmonar, hematológica, oncológica, assim como em pacientes que apresentam dor, desnutrição e manifestações psicológicas como a ansiedade e depressão, o que também corrobora com o outro FR encontrado neste estudo, o grupo de doenças ${ }^{(27)}$.

Outros fatores que podem contribuir para o aparecimento da fadiga são a idade e o número de

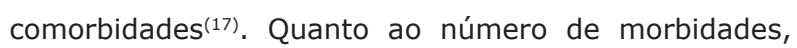
estudos mostraram associação entre o maior número de morbidades e a maior percepção de fadiga(28-29). Nos idosos, a fadiga se dá devido às alterações do organismo como um todo e do sistema cardiopulmonar, em que se observam a redução da captação de oxigênio, a redução da força dos músculos respiratórios e o aumento da resistência vascular. No sistema muscular ocorre diminuição da força e da flexibilidade muscular, resultando em fadiga, o que repercute nas atividades simples do cotidiano de idosos ${ }^{(30)}$.

Um estudo recente, realizado também em uma UTI do município de Ribeirão Preto, SP, com 626 pacientes adultos, mostrou que o FR fadiga apresentou maior sensibilidade para o diagnóstico PRI (23). Os autores enfatizam que pacientes com PRI com CD relacionadas à disfunção ventilatória, se não tratados adequadamente, esse diagnóstico pode evoluir para o diagnóstico de 
ventilação espontânea prejudicada (VEP), caracterizando um pior prognóstico do paciente ${ }^{(23)}$.

A idade avançada foi o segundo fator preditor de PRI neste estudo. O envelhecimento é caracterizado pela diminuição crônica da função do sistema orgânico, deixando o idoso susceptível a doenças, podendo desencadear o diagnóstico $\mathrm{PRI}^{(2)}$. O envelhecimento acarreta mudanças fisiológicas como o comprometimento da eficiência das trocas gasosas, a redução da complacência pulmonar, a diminuição da força dos músculos respiratórios e diminuição do transporte de oxigênio para os tecidos, com consequente diminuição do débito cardíaco, da massa muscular corpórea, do volume alveolar e da relação ventilação/perfusão, o que pode ocasionar o aparecimento do diagnóstico de enfermagem $\mathrm{PRI}^{(20)}$.

Neste sentido, cabe aos enfermeiros reconhecer essas peculiaridades e alterações durante a avaliação do exame físico e selecionar intervenções que melhorem o estado respiratório dentro do esperado para a idade. Estudo transversal realizado no Rio Grande do Sul, Brasil, identificou que quase metade dos idosos $(42,0 \%)$ apresentavam o diagnóstico PRI ${ }^{(31)}$. Nesta pesquisa, $86,7 \%$ dos pacientes idosos apresentaram PRI. Ressalta-se ainda que este grupo de pacientes é mais vulnerável à gripe, devido à maior prevalência de doenças crônico-degenerativas e ao comprometimento imunológico, o que pode causar alterações respiratórias e a manifestação de $\mathrm{PRI}^{(31-34)}$.

O terceiro fator preditor para PRI encontrado foi o grupo de doenças (trauma, cardiocirculatório e outros diagnósticos). As causas externas, como importante causador dos traumas, representado pelos acidentes de trânsito, representam um grave problema de saúde pública no Brasil, responsáveis por alta morbimortalidade, incapacidades, sequelas e considerável custo econômico ${ }^{(35)}$. Estudo que analisou 406 vítimas de trauma na cidade de São Paulo, SP, Brasil, identificou prevalência de $82,8 \%$ de pacientes com PRI (5).

Outro estudo realizado em uma unidade de urgência e emergência de um hospital público de grande porte no sul do Brasil identificou prevalência de 51,2\% de PRI em pacientes que foram vítimas de múltiplos traumas, sendo os principais $\mathrm{FR}$ a dor, o dano músculo esquelético, a hiperventilação e a disfunção neuromuscular e as principais CD a taquipneia e a bradipneia ${ }^{(36)}$. A presença destes indicadores clínicos ocorre devido à alteração na expansão pulmonar secundária à alteração da caixa torácica, além de hipovolemia secundária à hemorragia e a hipóxia decorrentes da lesão traumática. Assim, o controle da oxigenação cerebral e a oferta de oxigênio para os demais órgãos do corpo são fundamentais no atendimento a estes pacientes, além do controle de sangramento. A falta de assistência direcionada para o atendimento respiratório levará o paciente a desenvolver PRI ${ }^{(36-37)}$.

Em relação ao grupo de doenças relacionadas ao sistema cardiocirculatório, sabe-se que os pacientes com estas comorbidades podem apresentar descompensação cardíaca e causar alterações hemodinâmicas dando origem ao diagnóstico PRI. Os pacientes com insuficiência cardíaca à esquerda, por exemplo, podem apresentar sinais e sintomas de congestão pulmonar, devido à insuficiência ventricular esquerda, evidenciando o diagnóstico de enfermagem PRI. Além disso, os pacientes com síndrome coronariana aguda têm como principal sintoma a precordialgia. A dor anginosa aciona as manifestações do sistema simpático que causam aumento da frequência cardíaca, respiratória, alterando a mecânica respiratória quanto à profundidade, número de incursões por minuto e, se não tiver intervenção, levará à fadiga da musculatura respiratória e consequentemente, levando o paciente ao diagnóstico PRI ${ }^{(17)}$.

Assim, ao analisar o modelo final da regressão logística dos fatores preditivos de PRI, a curva apresentou uma boa capacidade preditiva das probabilidades de ocorrência de PRI (curva ROC 0,875), com alta sensibilidade e especificidade para identificação deste diagnóstico de enfermagem.

Outros fatores relacionados (obesidade e secreção brônquica), apesar de não serem identificados como preditores para PRI em nosso estudo, também se associaram a este diagnóstico. Observou-se também que as CDs alteração na profundidade respiratória, ausculta com ruídos adventícios, dispneia, murmúrios vesiculares diminuídos, taquipneia, tosse e uso da musculatura acessória para respirar também se associaram ao PRI ${ }^{(12-14,19)}$.

Nos indivíduos obesos, o diagnóstico PRI é identificado devido a redução do volume e capacidade pulmonar nestes pacientes. $O$ excesso de tecido adiposo provoca compressão mecânica do diafragma resultando em insuficiência respiratória restritiva, diminuição da complacência pulmonar e aumento da resistência pulmonar, o que consequentemente aumenta o trabalho respiratório e o consumo de oxigênio, resultando no diagnóstico PRI (38-39).

O FR secreção brônquica possivelmente se relacionou com o diagnóstico de enfermagem PRI devido ao estreitamento do lúmen consequente à produção exacerbada de secreções e também, devido a inabilidade dos pacientes de terapia intensiva em mobilizar ou expulsar as secreções espontaneamente do 
trato respiratório, conduzindo à dificuldade respiratória e ao diagnóstico PRI (12).

As CDs dispneia, taquipneia, alteração da profundidade respiratória e uso da musculatura acessória para respirar são alterações muito comuns em pacientes com PRI devido à fraqueza muscular respiratória oriunda da não resolução do problema de base que originou a descompensação respiratória. A taquipneia é o resultado da hiperventilação pulmonar, a qual se desenvolve como uma tentativa adaptativa de compensação(18). O fracasso desse mecanismo compensatório e o desequilíbrio entre a demanda e o fornecimento de oxigênio favorecem o aparecemimento do diagnóstico $\mathrm{PRI}^{(40-41)}$. O uso da musculatura acessória demonstra a tentativa de restabelecer um padrão respiratório normal. Um estudo realizado na cidade de Fortaleza, CE, Brasil, identificou que o uso da musculatura acessória aumenta em sete vezes a chance de ter o diagnóstico PRI ${ }^{(15)}$.

A tosse constitui um sintoma de uma grande variedade de patologias pulmonares e extrapulmonares, muito prevalente na população e produz impacto social negativo, intolerância no trabalho e na família, além de gerar grande custo em exames subsidiários e medicamentos. As maiores causas de tosse são as infecções virais das vias aéreas superiores (resfriado comum), das vias aéreas inferiores (traqueobronquites agudas), sinusites agudas, exposição a alérgenos e irritantes e exacerbações de doenças crônicas como a asma, a doença pulmonar obstrutiva crônica (DPOC) e as rinossinusites ${ }^{(42)}$, doenças estas que podem causar alterações da ventilação pulmonar, levando o indivíduo a apresentar o diagnóstico PRI. Um estudo transversal realizado em Fortaleza, $C E$, Brasil mostrou que o diagnóstico PRI foi o mais prevalente e as CDs mais encontradas foram os ruídos respiratórios adventícios e a tosse ${ }^{(43)}$. Os ruídos respiratórios adventícios são detectados na ausculta pulmonar e são frequentes em pacientes com alterações respiratórias em UTI(12).

Ao avaliar a especificidade, sensibilidade e valores preditivos positivos e negativos das CD e FR, observouse que a $C D$ murmúrios vesiculares diminuídos apresentou associação e excelente medida de acurácia, apresentando sensibilidade, especificidade, valor preditivo positivo e negativo altos para o diagnóstico de enfermagem PRI.

Apesar da sua importância, a CD murmúrio vesicular diminuído não consta na classificação da NANDA-I para este diagnóstico de enfermagem. Os murmúrios vesiculares são sons normais auscultados nas áreas pulmonares e sua diminuição é patológica e pode indicar desde a presença de atelectasias até diminuição da expansibilidade pulmonar(44). A atelectasia é uma complicação respiratória decorrente da obstrução de um brônquio, ou pulmão, por secreção ou corpo sólido, impedindo a passagem do ar e levando à diminuição do número de alvéolos funcionantes (44). Quando há obstrução completa em um brônquio que supre de ar uma região normalmente ventilada do parênquima pulmonar, o gás nos alvéolos distais à obstrução é absorvido na circulação pulmonar. Uma vez que todo o gás alveolar tenha sido absorvido na circulação, os alvéolos, agora sem gás, colabam, gerando a diminuição dos murmúrios vesiculares, causando alterações na ventilação respiratória e aparecimento do padrão respiratório ineficaz ${ }^{(44)}$.

Os resultados do estudo mostraram os fatores preditores para o diagnóstico de enfermagem PRI e as intervenções de enfermagem dedem ter um direcionamento precoce para os pacientes com fadiga, idade avançada, com grupo de doenças como o trauma, doenças cardiocirculatórias e outras doenças.

Como um fator positivo, esta pesquisa utilizou uma grande amostra de pacientes em estado crítico, uma medida objetiva de mensuração, a manovacuometria e contribuiu com novas CD e FR para o diagnóstico de Enfermagem PRI, o que proporcionará a melhoria da classificação de diagnósticos de enfermagem da NANDA Internacional, possibilitando um ensino de enfermagem mais preciso, além de trazer evidências para a prática clínica deste diagnóstico em enfermagem.

\section{Conclusão}

Os fatores preditores para PRI identificados foram a fadiga, idade avançada, traumas, doenças cardiocirculatórias e outras doenças. Ao analisar o modelo final por meio da curva ROC, observou-se que ele apresentou boa capacidade preditiva para PRI, associada a alta especificidade e sensibilidade. A CD murmúrios vesiculares diminuídos apresentou alta sensibilidade, especificidade e valores preditivos e negativos para PRI, demonstrando sua importância na identificação deste diagnóstico de enfermagem.

\section{Agradecimentos}

Aos funcionários da Unidade de Terapia Intensiva do Hospital de Urgências e Emergências de Rio Branco pela ajuda e disponibilidade em sua unidade para realização desta pesquisa.

\section{Referências}

1. Sarkar M, Madabhavi I, Niranjan N, Dogra M. Auscultation of the respiratory system. Ann Thorac Med. 2015;10(3):158-68. doi:10.4103/1817-1737.160831. 
2. Wuytack F, Meskell P, Conway A, McDaid F, Santesso N, Hickey FG, et al. The effectiveness of physiologically based early warning or track and trigger systems after triage in adult patients presenting to emergency departments: a systematic review. BMC Emerg Med. 2017;17:38. doi:10.1186/s12873-017-0148-z.

3. Kim MJ, Larson JL. Ineffective airway clearance and ineffective breathing patterns: Theorical and research base for nursing diagnosis. Nurs Clin North Am. [Internet]. 1987 Marc [Cited Jul 5, 2018];22(1):125-34. Available from: https://www.ncbi.nlm.nih.gov/pubmed/3644285 4. Herdman TH, Kamitsuru S. Diagnósticos de Enfermagem da NANDA: definições e classificação 2018-2020/ [NANDA Internacional]. 10a ed. Porto Alegre: Artmed; 2018.

5. Sallum AMC, Santos JLF, Lima FD. Nursing diagnoses in trauma victims with fatal outcomes in the emergency scenario. Rev. Latino-Am. Enfermagem. [Internet]. 2012 Feb [cited July 5, 2018]; 20(1):3-10. Available from: http://www.scielo.br/scielo.php?script=sci_ arttext\&pid=S0104-11692012000100002\&lng=en.

6. Okuno MFP, Costa N, Lopes MCBT, Campanharo CRV, Batista REA. The most used nursing diagnoses at an emergency service. Acta Paul Enferm. [Internet]. 2012 [cited July 5, 2018]; 25(2):256-62. Available from: http:// www.scielo.br/scielo.php?script=sci_arttext\&pid=S010321002012000200016\&lng=en.

7. Galdeano LE, Rossi LAR, Pezzuto TM. Nursing diagnosis of patients in the preoperatory period of cardiac surgery. Rev Esc Enferm USP. [Internet]. 2004 Sep [cited July 5, 2018]; 38(3): 307-16. Available from: http://www. scielo.br/scielo.php?script=sci_arttext\&pid=S008062342004000300009\&Ing=en.

8. Gordon M, Hiltunen E. High frequency: treatment priority Nursing diagnoses in critical care. Nurs Diagn. [Internet]. 1995 Oct-Dec [cited July 8, 2018];6(4):14354. Available from: https://www.ncbi.nlm.nih.gov/ pubmed/8573434

9. Araújo DS, Freire AF, Mendonça JKS, Bettencourt ARC, Amaral TLM, Prado PR. Construction and validation of a systematization instrument for nursing in intensive care. Rev Rene. 2015 Jul-Ago; 16(4):461-9. doi: 10.15253/2175-6783.2015000400002

10. Black LF, Hyatt RE. Maximal respiratory pressures: normal values and relationship to age and sex. Am Rev Respir Dis. 1969 May;99(5):696-702. doi: 10.1164/ arrd.1969.99.5.696

11. Souza RB. Sociedade Brasileira de Pneumologia e Tisiologia. Diretrizes para teste de função pulmonar. Pressões respiratórias estáticas máximas. Jornal Bras Pneumol. [Internet]. 2002 [cited July 9, 2018]; 28(3): S155-65. Available from: http://www.jornaldepneumologia. com.br/PDF/Suple_137_45_88\%20Press\%C3\%B5es\%20 respirat $\%$ C $3 \%$ B 3 rias $\% 20$ est $\%$ C $3 \%$ A 1 ticas $\% 20$ m\%C3\%A1ximas.pdf

12. Prado PR, Bettencourt ARC, Lopes JL. Defining characteristics and related factors of nursing diagnosis ineffective breathing pattern: na integrative literature review. Rev Bras Enferm. 2018. No prelo.

13. Avena M, Pedreira MLG, Gutiérrez MGR. Conceptual validation of the defining characteristics of respiratory nursing diagnoses in neonates. Acta Paul Enferm. [Internet]. 2014 Feb [cited July 9, 2018]; 27(1):76-85. Available from: http://www.scielo.br/scielo.php?script=sci arttext\&pid=S0103-21002014000100015\&lng=en. http:// dx.doi.org/10.1590/1982-0194201400015.

14. Cavalcante JCBC, Mendes LC, Lopes MVO, Lima $\mathrm{LH}$. Clinical indicators of ineffective breathing pattern in children with asthma. Rev RENE. [Internet]. 2010 Jan-Marc [cited July 9, 2018];11(1):66-75. Available from: http://www.revistarene.ufc.br/vol11n1_html_site/ a07v11n1.htm

15. Silva VM, Araujo TL, Lopes MVO. Evolution of nursing diagnoses for children with congenital heart disease. Rev. Latino-Am. Enfermagem [Internet]. 2006 Aug [cited 2018 Aug 24]; 14(4): 561-8. Available from: http:// www.scielo.br/scielo.php?script=sci_arttext\&pid=S010411692006000400014\&lng=en. http://dx.doi.org/10.1590/ S0104-11692006000400014.

16. Bertoncello KCG, Cavalcanti CDK, Ilha P. Real diagnoses and nursing intervention proposals for multiple trauma victims. Rev Eletron Enferm. [Internet]. 2013 Out/Dez;15(4):905-14. Disponível em: http://dx.doi. org/10.5216/ree.v15i4.19497.

17. Canto DF, Almeida MA. Nursing outcomes for ineffective breathing patterns and impaired spontaneous ventilation in intensive care. Rev Gaúcha Enferm. [Internet]. 2013 Dec [cited 2018 Aug 24] ; 34(4): 137-45. Available from: http://www.scielo.br/scielo.php?script=sci_ arttext\&pid=S1983-14472013000400018\&Ing=en.

18. Santos NA, Cavalcante TF, Lopes MVO, Gomes EB, Oliveira $\mathrm{C}$. Profile of nursing diagnoses in patients with respiratory disorders. Invest Educ Enferm. 2015; 33(1): 112-8. doi: 10.1590/S0120-53072015000100013.

19. Mota DDCF, da Cruz DALM, Pime CAM. Fatigue: a concept analyses. Acta Paul Enferm. [Internet]. 2005 Sep [cited July 9, 2018] ; 18(3): 285-93. Available from: http://www.scielo.br/scielo.php?script=sci_ arttext\&pid=S0103-21002005000300009\&Ing=en.

20. Ferreira EVM. Respiratory muscles: myths and secrets. J Bras Pneumol. [Internet]. 2015 Apr [cited July 9, 2018]; 41(2): 107-9. Available from: http:// www.scielo.br/scielo.php?script=sci_arttext\&pid=S180637132015000200107\&lng=en. http://dx.doi.org/10.1590/ S1806-37132015000200002. 
21. Silva GA. Obesity hypoventilation syndrome. Medicina. (Ribeirão Preto). [Internet]. 2006 Abr./Jun. [cited July 9, 2018]; 39(2):195-204. Available from: http://sobrafir.com.br/imagens_up/artigos/Obesidade_e_ Hipoventilacao_Alveolar.p

22. Caruso $P$, Albuquerque ALP, Santana PV, Cardenas LZ, Ferreira JG, Prina E, et al. Diagnostic methods to assess inspiratory and expiratory muscle strength. 2015 Mar-Apr;41(2):110-23. doi: 10.1590/S180637132015000004474.

23. Seganfredo $D H$, Beltrão BA, Silva VM, Lopes MVO, Castro SMJ, Almeida MA. Analysis of ineffective breathing pattern and impaired spontaneous ventilation of adults with oxygen therapy Rev. LatinoAm. Enfermagem. [Internet]. 2017 [cited July 9, 2018]; 25: e2954. Available from: http://www. scielo.br/scielo.php?script=sci_arttext\&pid=S0104$11692017000100395 \&$ Ing=en. Epub Dec 4, 2017. http://dx.doi.org/10.1590/1518-8345.1950.2954.

24. Ali NA, O'Brien JM Jr, Hoffmann SP, Phillips G, Garland A, Finley JC, et al. \& Midwest Critical Care Consortium. Acquired weakness, handgrip strength, and mortality in critically ill patients. Am J Respir Crit Care Med. 2008 Aug 1;178(3):261-8. doi: 10.1164/rccm.200712-18290C.

25. Maramattom BV, Wijdicks EF. Acute neuromuscular weakness in the intensive care unit. Critical Care Medicine. Crit Care Med. 2006 Nov;34(11):2835-41. doi: 10.1097/01.CCM.0000239436.63452.81

26. Khan J, Harrison TB, Rich MM. Mechanisms of neuromuscular dysfunction in critical illness. Crit Care Clin. 2008 Jan; 24(1): 165-x. doi: 10.1016/j. ccc.2007.10.004

27. Truong AD, Fan E, Brower RG, Needham DM. Benchtobeside review: mobilizing patients in the intensive care unit from pathophysiology to clinical trials. Crit Care. 2009 July; 13(4):216. doi: 10.1186/cc7885.

28. Latronico N, Herridge M, Hopkins RO, Angus D, Hart N, Hermans G, et al. The ICM research agenda on intensive care unit-acquired weakness. Intensive Care medicine. 2017 sep; 43(9):1270-81. doi: 10.1007/ s00134-017-4757-5

29. Castell MV, Sânchez M, Julián R, Queipo R, Martín $S$, Otero A. Frailty prevalence and slow walking speed in persons age 65 and older: implications for primary care. BMC Fam Pract. 2013 Jun 19;14:86. doi: 10.1186/14712296-14-86.

30. Neri AL, Yassuda MA, Araújo LF, Eulálio MC, Cabral BE, Siqueira MEC, et al. Methodology and social, demographic, cognitive, and frailty profiles of community-dwelling elderly from seven Brazilian cities: the FIBRA Study. Cad Saúde Pública. [Internet]. 2013 Apr [cited July 9, 2018]; 29(4):778-92. Available from: http://www. scielo.br/scielo.php?script=sci_arttext\&pid=S0102-
311X2013000400015\&lng=en. http://dx.doi.org/10.1590/ S0102-311X2013000400015.

31. Lira LN, Santos SSC, Vidal DAS, Gautério DP, Tomaschewski-Barlem JG, Piexak DR. Nursing diagnosis and prescriptions for hospitalized elderly. Av Enferm. [Internet]. 2015 May [cited July 9, 2018]; 33(2): 251-60. Available from: http://www. scielo.org.co/scielo.php?script=sci_arttext\&pid $=$ S0121$45002015000200007 \&$ Ing=en. http://dx.doi. org/10.15446/av.enferm.v33n2.30762.

32. Talbot HK. Influenza in older Adults. Infect Dis Clin North Am. 2017 Dec;31(4):757-66. doi: 10.1016/j. idc. 2017.07.005.

33. Stockton J, Stephenson I, Fleming D, Zambon M. Human metapneumovirus as a cause of communityacquired respiratory illness. Emerg Infect Dis. 2002 Sep;8(9):897-901. 10.3201/eid0809.020084

34. Boivin G, Abed Y, Pelletier G, Ruel L, Moisan D, Côté $S$, et al. Virological features and clinical manifestations associated with human metapneumovirus: a new paramyxovirus responsible for acute respiratory-tract infections in all age groups. J Infect Dis. 2002 Nov 1;186(9):1330-4. doi:10.1086/344319

35. Santos ZM: Oliveira ML. Assessment of knowledge, attitudes and practices of the elderly about the vaccine against Influenza in a Public Health Unit, Taguatinga, Federal District, Brazil, 2009. Epidemiol Serv Saúde. 2010 Sep; 19(3):205-16. doi: 10.5123/S167949742010000300003

36. Malvestio MAA, Sousa RMC. Survival after motor vehicle crash: impact of clinical and prehospital variables. Rev Saúde Pública. 2008 Aug; 42(4):639-47. doi: 10.1590/S0034-89102008000400009

37. ATLS Subcommittee; American College of Surgeons' Committee on Trauma; International ATLS working group. Adv Trauma Life Support (ATLS ${ }^{\circledR}$ ): the ninth edition. J Trauma Acute Care Surg. [Internet]. 2013 May [cited July 9, 2018]; 74(5):1363-6. Available from: http:// dx.doi.org/10.1097/TA.0b013e31828b82f5

38. Dal Sasso GTM, Barra DCC, Paese F, Almeida SRW, Rios GC, Marinho MM, et al. Computerized nursing process: methodology to establish associations between clinical assessment, diagnosis, interventions, and outcomes. Rev Esc Enferm USP. [Internet]. 2013 Feb [cited July 9, 2018]; 47(1): 242-9. Available from: http://www.scielo.br/scielo.php?script $=$ sci_ arttext\&pid=S0080-62342013000100031\&lng=en. http://dx.doi.org/10.1590/S0080-62342013000100031. 39. Schmidt M, Demoule A, Polito A, Porchet R, Aboab J, Siami $S$, et al. Dyspnea in mechanically ventilated critically ill patients. Crit Care Med. 2011 Sep;39(9):205965. doi: 10.1097/CCM.0b013e31821e8779. 
40. Goodridge D, Duggleby W, Gjevre J, Rennie D. Exploring the quality of dying of patients with chronic obstructive pulmonary disease in the intensive care unit: a mixed methods study. Nurs Crit Care. 2009 Mar-Apr;14(2):51-60. doi: 10.1111/j.14785153.2008.00313.x.

41. Sociedade Brasileira de Pneumologia e Tisiologia. IV Diretrizes brasileiras no manejo da asma. J Bras Pneumol. [Internet]. 2006 Nov [cited July 9, 2018]; 32(Suppl 7): S447-S74. Available from: http://www. scielo.br/scielo.php?script=sci_arttext\&pid=S180637132006001100002\&lng=en. http://dx.doi.org/10.1590/ S1806-37132006001100002

42. Pratter MR, Brightling CE, Boulet LP, Irwin RS. An empiric integrative approach to the management of cough: ACCP evidence-based clinical practice guidelines. Chest. 2006 Jan;129(1 Suppl):222S-31S. doi: 10.1378/ chest.129.1_suppl.222S.

43. Silveira UA, Lima LHO, Lopes MVO. Defined characteristics of the nursing diagnoses ineffective airway clearance and ineffective breathing pattern in asthmatic children. Rev RENE. 2008 out/dez; 9(4):125-33. doi: 10.15253/rev\%20rene.v9i4.5248

44. Cunha CS, Toledo RV. The Performance of the Physiotherapy in the Reversion of the Atelectasis: A report of a case in the Intensive Therapy Unit. Cads Unifoa. [Internet]. 2007 Aug [cited July 9, 2018]; 2(2):81-87. Available from: http://revistas.unifoa.edu.br/index.php/ cadernos/article/view/881 